\title{
Decreased white matter integrity before the onset of delusions in patients with Alzheimer's disease: diffusion tensor imaging
}

This article was published in the following Dove Press journal:

Neuropsychiatric Disease and Treatment

21 December 2012

Number of times this article has been viewed

\author{
Shutaro Nakaaki' \\ Junko Sato ${ }^{2}$ \\ Katsuyoshi Torii \\ Mizuki Oka' \\ Atsushi $\mathrm{Negi}^{2}$ \\ Takashi Nakamae ${ }^{3}$ \\ Jin Narumoto 3 \\ Jun Miyata ${ }^{4}$ \\ Toshi A Furukawa ${ }^{5}$ \\ Masaru Mimura' \\ 'Department of Neuropsychiatry, \\ Keio University School of Medicine, \\ Tokyo, Japan; ${ }^{2}$ Department of \\ Psychiatry and Cognitive-Behavioral \\ Medicine, Nagoya City University \\ Graduate School of Medical Sciences, \\ Nagoya, Japan; ${ }^{3}$ Department of \\ Psychiatry, Kyoto Prefectural \\ University of Medicine, Kyoto, Japan; \\ ${ }^{4}$ Department of Neuropsychiatry, \\ Kyoto University, Kyoto, Japan; \\ ${ }^{5}$ Department of Health Promotion \\ and Human Behavior (Cognitive- \\ Behavioral Medicine), Kyoto \\ University Graduate School of \\ Medicine/School of Public Health, \\ Kyoto, Japan
}

Correspondence: Shutaro Nakaaki Department of Neuropsychiatry, Keio University School of Medicine, 35 Shinanomachi, Shinjuku-ku,

Tokyo 160-8582, Japan

Tel +8I 353633829

Fax +8I 353790187

Email hzi05510@nifty.com
Background: The pathology of delusions in patients with Alzheimer's disease (AD) associated with white matter (WM) abnormalities is poorly understood. In addition, whether the abnormalities in WM integrity that underlie the delusions develop before the onset of the delusions remains unclear. In this study, we used a diffusion tensor imaging approach to examine the existence of baseline abnormalities in WM integrity in AD patients who developed delusions and $\mathrm{AD}$ patients who did not develop delusions.

Methods: Using the Neuropsychiatric Inventory, we identified patients with AD who exhibit delusions during a 1-year period. All the patients underwent a magnetic resonance imaging (MRI) examination at baseline. We conducted fractional anisotropy using tract-based spatial statistics software and compared the results of $\mathrm{AD}$ patients who developed delusions with those who did not develop delusions.

Results: Compared with the AD patients who did not develop delusions $(\mathrm{n}=15)$, the AD patients who developed delusions $(\mathrm{n}=10)$ exhibited two relatively large clusters and one minimal cluster of significantly lower fractional anisotropy results. The first cluster was located in the left parieto-occipital region and included several fibers: the left inferior longitudinal fasciculus, the inferior fronto-occipital fasciculus, the posterior corona radiate, and the forceps major of the corpus callosum. The second cluster was located on the body of the corpus callosum. A third minimal cluster was located on the superior temporal gyrus white matter.

Conclusion: Abnormalities in WM integrity involving several fibers may be crucial to the development of delusions in AD patients.

Keywords: Alzheimer's disease, white matter, DTI, delusions, tract-based spatial statistics

\section{Introduction}

Recent magnetic resonance imaging (MRI) studies have revealed that many patients with Alzheimer's disease (AD) have coexisting white matter hyperintensities. Although the significance of white matter hyperintensities in AD patients is poorly understood, several studies have suggested that the existence of white matter hyperintensities is associated with specific neuropsychiatric symptoms, such as delusions and nighttime disturbances. ${ }^{1-3}$ Furthermore, delusions, unlike other neuropsychiatric symptoms, are known to be associated with a poor prognosis. ${ }^{4}$

Diffusion tensor imaging (DTI), a relatively new MRI imaging procedure, has been developed to examine the integrity of white matter (WM) fiber bundles. The DTI technique has clarified that WM abnormalities are widespread throughout WM fiber bundles in AD patients. ${ }^{5}$ Fractional anisotropy (FA) is a widely used metric in studies of AD patients. Two reports have indicated that a reduction in 
FA in specific fiber tracts occurs during the early stage of AD. ${ }^{6,7}$ Together, these findings suggest that some neuropsychiatric symptoms, such as delusions, in patients with AD may be associated with a reduction in FA in several fiber bundles.

A 2-year prospective longitudinal study in AD patients has reported that the cumulative incidence of psychotic symptoms (delusions, hallucinations) was $36.9 \%{ }^{8}$ This study suggested that not all AD patients develop psychotic symptoms during the course of AD. Whether the baseline FA value in AD patients without delusions can predict future progression to delusions is a clinically important issue. Thus, we hypothesized that FA abnormalities may exist prior to the onset of delusions in AD patients who subsequently develop delusions. We performed DTI using baseline data for AD patients without delusions who were enrolled in a 1-year prospective longitudinal study. Then, after the 1-year follow-up period, we compared the FA values between $\mathrm{AD}$ patients who developed delusions and the AD patients who did not develop delusions, using tractbased spatial statistics.

\section{Methods}

\section{Participants}

The baseline sample consisted of Japanese AD patients with mild functional severity who attended the outpatient clinic of Nagoya City University Hospital between June 2009 and December 2010. The study inclusion criteria were (1) a diagnosis of probable AD according to the National Institute of Neurological and Communication Disorders and Stroke/Alzheimer Disease and Related Disorders Association criteria, ${ }^{9}(2)$ no history of medication with antipsychotic medications, and (3) no psychosis symptoms, as assessed using the Japanese version of the Neuropsychiatric Inventory (NPI). ${ }^{10,11}$ The NPI is a semiquantitative assessment based on information provided by the caregiver. The questionnaire consists of ten behavior-related questions concerning delusions, hallucinations, depression, anxiety, agitation, disinhibition, euphoria, irritability, apathy, and aberrant motor activity. The score for each item is obtained by multiplying the severity with the frequency. The total score is 120 . In accordance with our previous study, ${ }^{12}$ the psychosis symptoms were defined as delusions, hallucinations, agitation, disinhibition, irritability, and aberrant motor activity.

In 2011, the National Institute on Aging and the Alzheimer's Association proposed a revision of the criteria for AD. ${ }^{13}$ However, we started this study in June 2009. The revised criteria require the examination of multiple biomarkers, including the need for a positron emission tomography (PET) examination, and the widespread clinical adoption of the revised criteria remains controversial. ${ }^{14}$ Thus, we did not adopt the revised criteria that were updated in 2011.

Patients were excluded if (1) other neurological diseases were present, (2) the patient had a previous history of mental illness or substance abuse before the onset of dementia, (3) either an MRI or a CT scan had revealed focal brain lesions, (4) the patient's Mini-Mental State Examination (MMSE) $)^{15}$ score was less than 11 , or (5) reliable informed consent could not be obtained from the patient and/or his/her relatives. The study protocol was approved by the Ethics Committee of Nagoya City University Medical School. Both the subjects and the caregivers were informed of the purpose and procedures of this study and were asked to sign a consent form.

\section{Follow-up assessment}

The follow-up assessment was conducted within one year after the baseline assessment. The presence of delusions was diagnosed based on the delusion score of the NPI. In agreement with previous studies, ${ }^{8}$ a score of greater than 3 was regarded as indicating the presence of delusions. An independent psychiatrist who was unaware of the MRI results conducted the NPI evaluation. Both the MMSE and the NPI were assessed at 3-month intervals from the baseline examination.

\section{MRI image acquisition}

DTI data were acquired using single-shot, spin-echo echoplanar sequences on a 1.5-T MRI system (Gyoroscan Intera; Royal Philips Electronics, Amsterdam, The Netherlands) with a 33- $\mathrm{m} \mathrm{Tm}^{-1}$ gradient and a receiver-only, six-channel phasedarray head coil. The scanning parameters were as follows: echo time $=80 \mathrm{~ms}$; repetition time $=6000 \mathrm{~ms} ; 128 \times 128$ matrices, field of view $=224 \times 224 \mathrm{~mm}^{2}, 45$ continuous axial slices of $3.0 \mathrm{~mm}$ thickness, 15 noncollinear axis motion probing gradient, $\mathrm{b}=800 \mathrm{~s} \cdot \mathrm{mm}^{-2}$. Scanning was repeated two times to enhance the signal-to-noise ratio. The MRI studies were performed in the Department of Radiology of Nagoya City University Hospital. The MRI examination was conducted at baseline.

\section{Data processing and analysis}

DTI data processing was performed using FSL Ver 4.1 (Neuroimaging Informatics Tools and Research Clearinghouse [NITRC], Washington, DC, USA). All the data sources were corrected for eddy currents and head motion 
by registering all the data with the first $\mathrm{b}=0$ image of the first repetition, using affine transformation. A voxel-wise statistical analysis of the FA data was performed using tract-based spatial statistics (FMRIB, Oxford, UK). ${ }^{16}$ FA maps were calculated using the FMRIB's Diffusion Tool program. Both the AD patients who developed delusions and the AD patients who did not develop delusions were tested with 10,000 permutations. The statistical threshold was set at $P<0.05$, and the results were corrected for multiple comparisons using threshold-free cluster enhancement. ${ }^{16}$ Threshold-free cluster enhancement produces an output image in which the voxel value represents the weighed sum of the local clustered signal. We performed group comparisons using an analysis of covariates with age and gender, and MMSE score as nuisance covariates.

\section{Results}

Twenty-eight AD patients were enrolled in this study. Three AD patients had been lost to follow up at the end of the 1-year period; at the 1-year follow-up examinations, the remaining $25 \mathrm{AD}$ patients had completed the study. Among these patients, ten AD patients had developed delusions, and the remaining $15 \mathrm{AD}$ patients had not developed delusions during the follow-up period. The types of delusions in the AD patients, as identified by the NPI, were persecutory delusions $(n=9)$ and misidentification delusions $(n=1)$. The mean time until the development of delusions from the baseline assessment was 7.2 months (minimum of 3 months, maximum of 12 months).

The demographic data at baseline for the AD patients are summarized in Table 1. No significant differences in the

Table I Demographic data of AD patients who did or did not develop delusions

\begin{tabular}{lcc}
\hline & \multicolumn{2}{c}{ Patients with Alzheimer's disease } \\
\cline { 2 - 3 } & $\begin{array}{l}\text { With delusion } \\
(\mathbf{n}=\mathbf{I 0})\end{array}$ & $\begin{array}{l}\text { Without delusion } \\
(\mathbf{n}=\mathbf{I 5})\end{array}$ \\
\hline Male/female & $\mathrm{I} / 9$ & $2 / 13$ \\
Age, years & $79.2 \pm 5.9$ & $76.3 \pm 7.6$ \\
Education, years & $9.1 \pm 2.2$ & $10.9 \pm 2.7$ \\
Duration of illness, years & $1.6 \pm 0.9$ & $1.8 \pm 0.3$ \\
MMSE score & $19.8 \pm 2.1$ & $2 \mathrm{I} .1 \pm 1.4$ \\
NPI & & \\
Depression/dysphoria & $0.3 \pm 0.4$ & $0.2 \pm 0.4$ \\
Apathy & $2.7 \pm 1.3$ & $3.0 \pm 1.1$ \\
\hline
\end{tabular}

Notes: Data are presented as the mean \pm SD. No significant differences in any of the items were observed between AD patients who did and those who did not develop delusions.

Abbreviations: AD, Alzheimer's disease; MMSE, Mini-Mental State Examination; NPI, Neuropsychiatric Inventory. demographic variables were found between the AD patients who developed delusions $(n=10)$ and those who did not develop delusions $(n=15)$.

Regarding the NPI subscale scores, with the exception of depression/dysphoria and apathy, the two groups of AD patients both scored 0 at baseline. Regarding the depression/ dysphoria and apathy subscales, no significant differences were found between the two groups of AD patients (Table 1). During the 1-year study period, no significant differences in the depression/dysphoria and apathy subscales were found between the two groups of $\mathrm{AD}$ patients at the time of the appearance of delusions (depression/dysphoria: AD patients with delusions, $0.4 \pm 0.5$; AD patients without delusions, $0.3 \pm 0.4$; apathy: AD patients with delusions, $3.5 \pm 1.6$; AD patients without delusions, $3.9 \pm 1.9$ ). However, during the 1-year study period, the AD patients with delusions had significantly higher scores $(P<0.01)$ for both agitation and irritability on the NPI than did the AD patients without delusions, at the time of the appearance of delusions (agitation: AD patients with delusions, $2.4 \pm 01.1$; AD patients without delusions, $0.1 \pm 0.3$; irritability: AD patients with delusions, $2.5 \pm 0.9$; AD patients without delusions, $0.1 \pm 0.3$ ).

In the group comparison, the $\mathrm{AD}$ patients who developed delusions showed three clusters of significantly lower FA values compared with the AD patients who did not develop delusions. The first cluster (maximum cluster) was located in the left parieto-occipital region (peak MNI coordinates, $\mathrm{x}=-56, \mathrm{y}=-37, \mathrm{z}=25$; cluster size $=3741)$ (Figure 1).

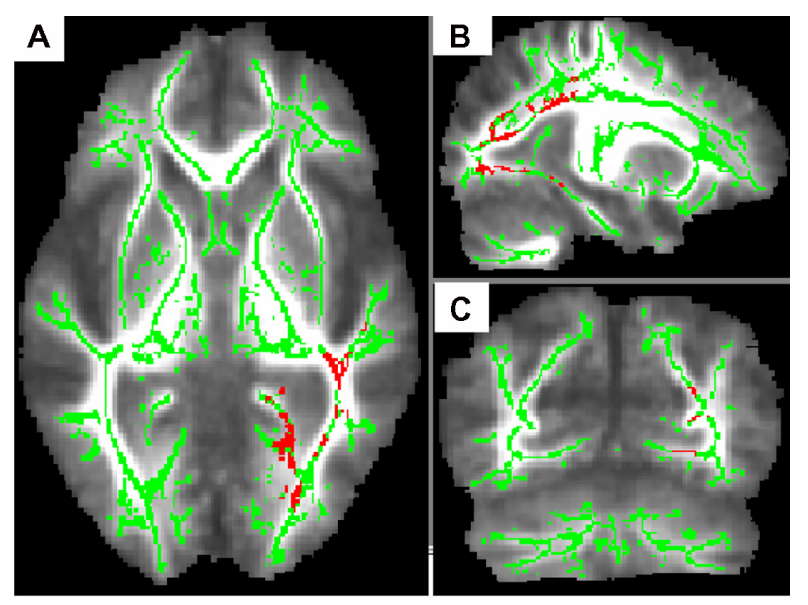

Figure I AD patients who developed delusions $(n=10)$ exhibited lower FA values than those who did not develop delusions $(n=15)$. To aid visualization, the regions with significant FA reductions ( $P<0.05$, corrected by TFCE) have been thickened (pink-red). The results (left inferior longitudinal fasciculus, left inferior fronto-occipital fasciculus, left posterior corona radiate, left forceps major of the corpus callosum) are shown overlaid on the mean FA map and the FA skeleton (green); (A) axial slice; (B) sagittal slice; (C) coronal slice.

Abbreviations: AD, Alzheimer's disease; FA, fractional anisotropy; TFCE, threshold-free cluster enhancement. 
These areas include several fibers, the left inferior longitudinal fasciculus (ILF), the left inferior fronto-occipital fasciculus (IFOF), the left posterior corona radiate, and the left forceps major of the corpus callosum. The second cluster was located on the body of the corpus callosum (CC) (peak MNI coordinates, $x=5, y=8, z=24$; cluster size $=935$ ). The third cluster (minimum cluster) was located on the right superior temporal gyrus white matter (peak MNI coordinates, $\mathrm{x}=43, \mathrm{y}=-30, \mathrm{z}=4$; cluster size $=58$ ).

\section{Discussion}

To our knowledge, this is the first DTI study to use tractbased spatial statistics to examine white matter abnormalities at baseline, between $\mathrm{AD}$ patients who developed delusions and those who did not develop delusions. We identified two relatively large clusters of reduced FA in AD patients who developed delusions: one in the left parieto-occipital region, and the other in the body of the corpus callosum. The former cluster contains multiple fiber tracts, such as the left ILF and the IFOF. Several recent studies have suggested that abnormally low FA exists in late-myelinating fibers, such as the ILF, IFOF, and CC, in AD patients. ${ }^{17,18}$ Thus, it is clinically important to note that these significant FA reductions were observed in the AD patients who developed delusions relative to what was observed in AD patients who did not develop delusions, in this study.

A few, but significant, neuroimaging studies have demonstrated that both attentional, visuoperceptual, and memory deficits contribute to delusions in AD patients. ${ }^{19,20}$ The widespread attentional network may play an important role in both anterior and posterior associations in the development of delusions. Both ILF and IFOF are large fibers connected to corticortical pathways. The former association fiber connects the occipital and temporal lobes, while the latter connects the occipital and frontal lobes. These fibers are thought to play a role in the emotional valence of visual processing. ${ }^{21,22}$ Recent studies ${ }^{23,24}$ have reported an association between delusion in $\mathrm{AD}$ and cognitive impairments such as visuospatial and executive functions. Unfortunately, we did not perform detailed neuropsychological tests other than the MMSE in the AD patients. No significant differences in the MMSE scores were found between the AD patients who developed delusions $(n=10)$ and those who did not develop delusions $(n=15)$. Thus, the association between detailed cognitive impairments and delusion in AD was unclear in this study. Further studies are needed to clarify the influence of neuropsychological function on AD patients who develop delusions.
The $\mathrm{CC}$ is the largest interhemispheric white matter commissure connecting the cerebral hemispheres. ${ }^{25}$ Thus, the breakdown of connectivity between widespread areas may contribute to the development of delusions. Our study indicated that $\mathrm{AD}$ patients who develop delusions may have vulnerable WM structures in several fiber tracts. Several previous functional neuroimaging studies (either single photon emission computed tomography [SPECT] or PET) have suggested that a right hemispheric pathology is associated with delusions in AD patients. ${ }^{26}$ The difference in laterality in this study (left dominance) may be attributed to methodological differences (ie, DTI analysis in this study vs functional neuroimaging in previous studies). In addition, recent studies using SPECT have demonstrated that the neural basis underlying persecutory delusions and misidentification delusions may differ in $\mathrm{AD}$ patients with delusions. Fukuhara et a ${ }^{20}$ reported that the regional cerebral blood flow in the right posterior parietal region was lower in AD patients with persecutory delusions than in AD patients without delusions. However, Nakano et $\mathrm{al}^{27}$ reported a lower regional cerebral blood flow in bilateral anterior cingulate gyri in $\mathrm{AD}$ patients with persecutory delusions compared with AD patients with misidentification delusions. In our study, the limited number of $\mathrm{AD}$ patients with misidentification delusions $(\mathrm{n}=1)$ did not allow such an analysis. Thus, we should be cautious when interpreting the findings of this study, since only a limited number of AD patients were included in this pilot study. DTI analyses in further longitudinal, large-sample studies of AD patients who develop delusions are needed to support the findings of the present study.

\section{Acknowledgments}

The authors gratefully acknowledge the grant from a Grant-in-Aid for Scientific Research (c) (22530750, 22591293) from the Ministry of Education, Culture, Sports, Sciences and Technology in Japan.

\section{Disclosure}

The authors report no conflicts of interest in this work.

\section{References}

1. Hirono N, Kitagaki H, Kazui H, Hashimoto M, Mori E. Impact of white matter changes on clinical manifestation of Alzheimer's disease: A quantitative study. Stroke. 2000;31(9):2182-2188.

2. Lee DY, Choo IH, Kim KW, et al. White matter changes associated with psychotic symptoms in Alzheimer's disease patients. J Neuropsychiatry Clin Neurosci. 2006;18(2):191-198.

3. Ogawa Y, Hashimoto M, Yatabe Y, et al. Association of cerebral small vessel disease with delusions in patients with Alzheimer's disease. Int J Geriatr Psychiatry. Epub March 7, 2012. 
4. Scarmeas N, Brandt J, Albert M, et al. Delusions and hallucinations are associated with worse outcome in Alzheimer disease. Arch Neurol. 2005;62(10):1601-1608

5. Oishi K, Mielke MM, Albert M, Lyketsos CG, Mori S. DTI analyses and clinical applications in Alzheimer's disease. J Alzheimers Dis. 2011;26 Suppl 3:287-296.

6. Zhang Y, Schuff N, Jahng GH, et al. Diffusion tensor imaging of cingulum fibers in mild cognitive impairment and Alzheimer disease. Neurology. 2007;68(1):13-19.

7. Mielke MM, Kozauer NA, Chan KC, et al. Regionally-specific diffusion tensor imaging in mild cognitive impairment and Alzheimer's disease. Neuroimage. 2009;46(1):47-55.

8. Aalten P, de Vugt ME, Lousberg R, et al. Behavioral problems in dementia: a factor analysis of the neuropsychiatric inventory. Dement Geriatr Cogn Disord. 2003;15(2):99-105.

9. McKhann G, Drachman D, Folstein M, Katzman R, Price D, Stadlan EM. Clinical diagnosis of Alzheimer's disease: report of the NINCDS-ADRDA Work Group under the auspices of Department of Health and Human Services Task Force on Alzheimer's Disease. Neurology. 1984;34(7):939-944.

10. Cummings JL, Mega M, Gray K, Rosenberg-Thompson S, Carusi DA, Gornbein J. The Neuropsychiatric Inventory: comprehensive assessment of psychopathology in dementia. Neurology. 1994;44(12): 2308-2314.

11. Hirono N, Mori E, Ikejiri Y, et al. Japanese version of the Neuropsychiatric Inventory - a scoring system for neuropsychiatric disturbance in dementia patients. No To Shinkei. 1997;49(3):266-271. Japanese.

12. Matsui T, Nakaaki S, Murata Y, et al. Determinants of the quality of life in Alzheimer's disease patients as assessed by the Japanese version of the Quality of Life-Alzheimer's disease scale. Dement Geriatr Cogn Disord. 2006;21(3):182-191.

13. McKhann GM, Knopman DS, Chertkow H, et al. The diagnosis of dementia due to Alzheimer's disease: recommendations from the National Institute on Aging-Alzheimer's Association workgroups on diagnostic guidelines for Alzheimer's disease. Alzheimers Dement. 2011;7(3):263-269.

14. Frisoni GB, Winblad B, O’Brien JT. Revised NIA-AA criteria for the diagnosis of Alzheimer's disease: a step forward but not yet ready for widespread clinical use. Int Psychogeriatrics. 2011;23(8):1191-1196.
15. Folstein MF, Folstein SE, McHugh PR. "Mini-mental state". A practical method for grading the cognitive state of patients for the clinician. J Psychiatr Res. 1975;12(3):189-198.

16. Smith SM, Nichols TE. Threshold-free cluster enhancement: addressing problems of smoothing, threshold dependence and localisation in cluster inference. Neuroimage. 2009;44(1):83-98.

17. Teipel SJ, Born C, Ewers M, et al. Multivariate deformation-based analysis of brain atrophy to predict Alzheimer's disease in mild cognitive impairment. Neuroimage. 2007;38(1):13-24.

18. Stricker NH, Schweinsburg BC, Delano-Wood L, et al. Decreased white matter integrity in late-myelinating fiber pathways in Alzheimer's disease supports retrogenesis. Neuroimage. 2009;45(1):10-16.

19. Staff RT, Shanks MF, Macintosh L, Pestell SJ, Gemmell HG, Venneri A. Delusions in Alzheimer's disease: spet evidence of right hemispheric dysfunction. Cortex. 1999;35(4):549-560.

20. Fukuhara R, Ikeda M, Nebu A, et al. Alteration of rCBF in Alzheimer's disease patients with delusions of theft. Neuroreport. 2001;12(11): 2473-2476.

21. Catani M, Jones DK, Donato R, Ffytche DH. Occipito-temporal connections in the human brain. Brain. 2003;126(Pt 9):2093-2107.

22. Miyata J, Yamada M, Namiki C, et al. Reduced white matter integrity as a neural correlate of social cognition deficits in schizophrenia. Schizophr Res. 2010;119(1-3):232-239.

23. Perez-Madriñan G, Cook SE, Saxton JA, et al. Alzheimer disease with psychosis: excess cognitive impairment is restricted to the misidentification subtype. Am J Geriatr Psychiatry. 2004;12(5):449-456.

24. Nagata T, Ishii K, Ito T, et al. Correlation between a reduction in Frontal Assessment Battery scores and delusional thoughts in patients with Alzheimer's disease. Psychiatry Clin Neurosci. 2009;63(4): 449-454.

25. Nakamae T, Narumoto J, Sakai Y, et al. Diffusion tensor imaging and tract-based spatial statistics in obsessive-compulsive disorder. J Psychiatr Res. 2011;45(5):687-690.

26. Ismail Z, Nguyen MQ, Fischer CE, Schweizer TA, Mulsant BH. Neuroimaging of delusions in Alzheimer's disease. Psychiatry Res. 2012;202(2):89-95.

27. Nakano S, Yamashita F, Matsuda H, Kodama C, Yamada T. Relationship between delusions and regional cerebral blood flow in Alzheimer's disease. Dement Geriatr Cogn Disord. 2006;21(1):16-21.
Neuropsychiatric Disease and Treatment

\section{Publish your work in this journal}

Neuropsychiatric Disease and Treatment is an international, peerreviewed journal of clinical therapeutics and pharmacology focusing on concise rapid reporting of clinical or pre-clinical studies on a range of neuropsychiatric and neurological disorders. This journa is indexed on PubMed Central, the 'PsycINFO' database and CAS.

\section{Dovepress}

The manuscript management system is completely online and includes a very quick and fair peer-review system, which is all easy to use. Visit http://www.dovepress.com/testimonials.php to read real quotes from published authors. 